\title{
Lightning Induced Voltages on Buried Shielded Cables
}

\author{
R. Suresh Babu and M. Joy Thomas \\ Department of High Voltage Engg, Indian Institute of Science \\ Bangalore - 560012 , INDIA
}

\begin{abstract}
This paper presents the horizontal electric fields computed at the ground surface and at different depths below the ground surface due to a nearby lightning stroke. Using the horizontal field thus calculated at various points, the induced voltage on a buried cable has been computed using a coupling equation, which incorporates the transfer impedance of the cable. The influence of ground parameters like conductivity and relative permittivity have been considered in the calculation of electric field and hence the induced voltages.
\end{abstract}

\section{INTRODUCTION}

Numerous outages and interference problems are encountered in overhead as well as underground power and communication systems due to the transient electromagnetic disturbances caused by a nearby lightning stroke. Lightning disturbances are largely atmospheric phenomena governed by physical properties of the air. The earth serves as a return conductor for the lightning currents and hence the effect of lightning on buried power and communication systems are primarily earth conduction problems caused by the finite conductivity of the ground. Current and voltage pulses could he induced on the buried cables running between two places, buildings or equipment enclosures and thereby enter the terminal equipments.

A significant amount of theoretical work has already been done on interaction of lightning electromagnetic fields with overhead power lines and low voltage power installations. These studies led to the development of mathematical tools for the calculations of lightning generated electromagnetic fields at different distances from the strike point and at different heights above the ground [1].

However, there are many situations, in which the low voltage power installations as well as communication and computer network cables are laid underground. The.underground installations are more immune to induction effects from the electromagnetic fields than the overhead ones. The sensitivity and the low threshold 'energies needed to destroy modem electronics call for a thorough investigation of induction effects in these installations. To facilitate such investigations a detailed description of lightning electromagnetic fields is required at different depths below the earth's surface.

In addition, lightning surge analysis is quite important for the design of protection circuit as well as insulation design in underground low voltage power distribution systems as well as communication and computer network cables. Hence there is a need for evaluation of lightning induced voltages on buried systems.

\section{PROBLEM GEOMETRY AND LIGHTNING CHANNEL MODEL}

Figure 1 represents the geometry of the present problem. The lightning strikes the ground near a buried cable. The lightning channel is assumed to he a straight and vertical antenna above the ground plane with no channel branches. The problem is to compute the overvoltages induced on the cable conductor at different points along the cable conductor, for a given channel base current (i.e., the current in the lightning channel at ground surface). It is proposed to study the influence of ground parameters, viz., conductivity and relative permittivity on the induced voltages.

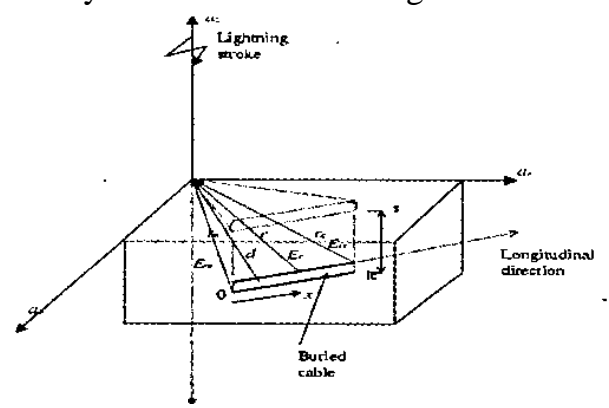

Figure 1. Geometry of the problem 


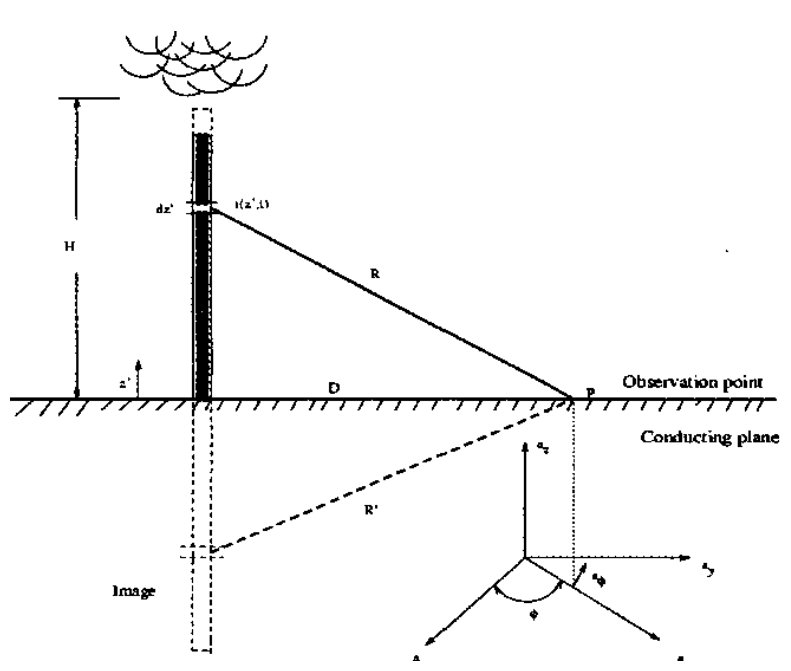

Figure 2. Illustration of geometrical parameters

In the present work modified transmission line model has been used for describing the lightning return stroke current, which is discussed briefly below In the MTL model [2] lightning current intensity is assumed to decrease exponentially while propagating up the channel and is expressed as follows

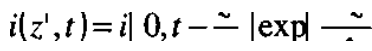

$i\left(z^{\prime}, t\right)=0 \quad z^{\prime} \geq v t$

where

$v \quad$ is the lightning return stroke velocity.

2 is the decay constant which allows the current to decrease its amplitude with height.

$i\left(z^{\prime}, t\right)$ is the current along the channel obtained from the return-stroke current model.

The geometrical parameters are shown in figure 2 .

\section{COMPUTATION OF INDUCED VOLTAGES}

\section{Computation of Surface Fields}

By assuming that the earth is flat and perfectly conducting, the electromagnetic field radiated by a lightning channel of infinitesimal length $\mathrm{dz}^{\prime}$ at height $z^{\prime}$ in time domain at a general point on the earth's surface at a distance ' $\mathrm{D}$ ' from the striking point can be calculated by employing the equations given below which are

$d E_{r}(D, \phi, z=0, t)=$ $\frac{d z^{\prime}}{4 \pi \varepsilon_{o}}\left|\begin{array}{l}\frac{3 D z^{\prime}}{R^{5}} \int_{0}^{1} i\left(z^{\prime}, \tau-\frac{R}{c}\right) \\ \frac{3 D z^{\prime}}{c^{2} R^{3} i\left(z^{\prime}, \tau-\frac{R}{c}\right)} \quad \text { at }\end{array}\right| a_{r}$

$d E_{z}(D, \phi, z=0, t)=$

$\frac{d z^{\prime}}{4 \pi \varepsilon_{o}}\left(\begin{array}{l}\frac{2 z^{2}-D^{2}}{R^{5}} \int_{0}^{1} i\left(z^{\prime}, \tau-\frac{R}{c}\right) d \tau+\frac{2 z^{\prime 2}-D^{2}}{c R^{4}} i\left(z^{\prime}, \tau-\frac{R}{c}\right)- \\ \frac{D^{2}}{c^{2} R^{3}} \frac{\partial i\left(z^{\prime}, \tau-\frac{R}{c}\right)}{\partial t}\end{array}\right) a_{z}$

$d B_{\phi}(D, \phi, z=0, t)=$

$\frac{\mu_{0} d z^{\prime}}{4 \pi}\left(\frac{D}{R^{3}} i\left(z^{\prime}, \tau-\frac{R}{c}\right)+\frac{D}{c R^{2}} \frac{\partial i\left(z^{\prime}, \tau-\frac{R}{c}\right)}{\partial t}\right) a_{\phi}$.

(5)

In equations ( 3 ) and (4), the first term is called the electrostatic field, the second, the electric induction or intermediate field, and the third the electric radiation field. In (5), the first term is called the magnetic induction and the second, the magnetic radiation field

The field produced by the return stroke is obtained by integrating (3-5) along the channel and its image.

It is generally accepted that, for the problems we are dealing with, the vertical and azimuthal magnetic fields are not much affected by the ground conductivity and thus they are generally calculated assuming ground as a perfectly conducting plane. In fact, the contribution of the source dipole and its image to these field components and consequently, small variations in the image field due to the finite ground conductivity will have little effect on the total fields. The above approximation is particularly reasonable at close distances from the channel (less than some kilometers) where as at larger distances, the propagation of the lightning fields on a real soil no longer can be neglected. The horizontal electric field component is markedly affected by the ground conductivity. Indeed for this component, the effects of the two contributions subtract, and small changes in the image field may lead to appreciable changes in the total horizontal electric field. 
If the conductivity of the ground is finite, the electromagnetic field equations become more complex and contain slowly converging integrals (Sommerfeld integrals). The problem of the radiation of a vertical dipole above a soil of a finite conductivity has been treated by Somerfeld who determined the solutions of Maxwell's equations taking into account the boundary conditions at the air-soil interface

This exact formulation, requires a large computational time when using it is as an input to transmission line coupling calculations. In order to reduce the computational time some approximations to the above approaches, are presented in the literature. Two of the mathematically simpler approximations, namely the Wave Tilt Formula and the Rubinstein approximation, are discussed below.

The wave tilt formula applies rigorously to the case of plane waves with grazing angle of incidence with respect to ground plane. It relates the Fourier transform of the horizontal electric field $E_{r, \sigma}(j \omega)$ and the vertical field $E_{z, \infty}(j \omega)$.

$$
W(j \omega)=\frac{E_{r, \sigma}(j \omega)}{E_{z, \omega}(j \omega)}=\frac{1}{\sqrt{\left(\varepsilon_{r}+\frac{\sigma_{g}}{j \omega \varepsilon_{0}}\right)}}
$$

where

$\infty \quad$ is the subscript that indicates the fields calculated assuming a perfect ground,

$\omega \quad$ is the angular frequency;

$\mathcal{\varepsilon}$ is the relative permittivity of the ground;

$\sigma_{g} \quad$ is the ground conductivity.

The approximation proposed by Rubinstein is given by the following expression

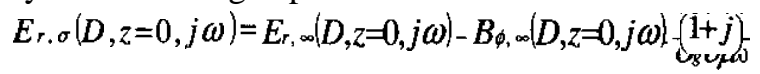

where

$B_{\phi, \infty}$ is the Fourier transform of azimuthal magnetic field at the ground level;

6 is the skin depth factor and is given by $\sqrt{\frac{2}{\omega \sigma_{\mathrm{s}} \mu_{\mathrm{s}}}}$

$\mu_{\mathrm{g}} \quad$ is the ground permeability.

$\sigma_{g} \quad$ is the ground conductivity

The basic assumptions in the Rubinstein approximation are that $\sigma_{g} \gg \omega_{\mathcal{B}} \varepsilon_{\mathrm{E}}$ and that the horizontal magnetic field and vertical electric field at ground level can be assumed to be equal to the values obtained for the case of a perfectly conducting ground plane. The two approximations mentioned above exhibit features that can be easily integrated in a time domain approach for coupling calculations, and do not need large computational time.

\section{Computation of Underground Fields}

The horizontal electric field is related to the azimuthal magnetic field through the surface impedance relationship. Thus, the azimuttal magnetic field at the surface $(s=0)$ is related to the horizontal electric field at the surface through the equation given by (7). This relationship can be used to obtain the horizontal electric field from the azimuthal magnetic field [3]. This is an advantage since the azimuthal magnetic field generated by return strokes can be measured without much difficulty.

The horizontal electric field penetrates the earth in exactly the same way as the magnetic field. Thus the horizontal field for a finitely conducting ground, $E_{r,} \sigma$, at depth 's' is given by[4]

$$
E_{r, \sigma}(D, s, t)=\int_{0} E_{r, \sigma}(D, 0, t-\tau) Y(\tau)
$$

with

$$
Y(t)=\frac{\exp \left(\frac{-a t}{2}\right) a k}{2 \sqrt{t^{2}-k^{2}}} I_{0}\left(\frac{a \sqrt{t^{2}-k^{2}}}{2}\right) u(t-k)+\exp \left(\frac{-a t}{2}\right) \delta(t-k)
$$

where

$a=\frac{\sigma_{s}}{\varepsilon_{a} \varepsilon_{r}}$

In these equations 6 is the delta function and $u(t)$ is the Heaviside's unit step function $E_{r, \sigma}(D, 0, t)$ is the horizontal electric field in time domain on the surface of the ground and $I_{o}$ is the zeroth order modified Bessel function of the first kind. The equation (8) completely specifies the underground fields in terms of the magnetic fields that are present at the surface. The surface fields can be easily measured, or, if the temporal and spatial variation of the return stroke current is known, they can easily be calculated.

\section{Coupling model}

For arriving at the coupling model, the case of a long buried cable passing through the influence zone of a 
nearby lightning channel has been considered. Even assuming a lightning return stroke current not directly terminating on the cable, the induced surges can be destructive to the communication cable. Special attention is, on the other hand, paid to the surge voltage, $V_{m}$ induced on the conductor internal to the shielded cable. This quantity is important for the insulation as well as surge protection design. A critical example is a communication cable with the nonmagnetic shield, covered by a protecting jacket. Under the same exposure conditions, the coupling to the'power cable is of minor importance. Infact, disregarding the saturation phenomena, the overall ferromagnetic armour causes the induced voltages to be remarkably attenuated. In any case, a power cable can be considered substantially immune to lightning failure because of the much heavier insulation.

The coupling mechanism for the cable takes into account the longitudinal component $E_{r x}$ of the undisturbed horizontal electric field $E_{r}$ applied along the laying line below ground. The model requires, in fact, a series voltage distribution, just equal to $E_{r x}$, which drives a transmission line representing the shield ground coaxial system. The well known coupled telegrapher's equation with incorporated series voltage per unit length allow the induced current $I_{s h}$ carried by the shield to be resolved with the use of Green's functions [6,7]. Thereby, an approximation to the induced inner voltage $V_{x}$, experienced by the core-shield insulation at the generic cable cross-section distance $\mathrm{x}$ from the origin (fig 2) can be calculated as follows:

$$
V_{m}=\frac{I_{s h}}{2} Z_{T}\left[x-\left(l_{c}-x\right)\right]
$$

where $Z_{T}$ denotes the transfer impedance and $l_{c}$ is the cable length. For a straight cable extending from $\mathrm{x}=0$ to $\mathrm{x}=l_{\mathrm{c}}$ the directional correlation between $E_{r}$ and $E_{r x}$ leads to

$$
I_{s h}=\frac{1}{2 Z_{g o}} \int_{r_{o}}^{r_{c}} E_{r} d r
$$

in which $Z_{g o}$ is the characteristic impedance of the shield coaxial line.

For nonmagnetic shield cable,

$$
Z_{\tau} \approx \frac{1}{2 \pi a \sigma_{c} \tau}
$$

$Z_{g o} \approx \frac{1}{2 \pi} \sqrt{\frac{10 \mu_{o}}{\varepsilon_{o} \varepsilon_{r c}} \ln (b / a)}$

have been assumed [8], where a, $\sigma_{c}$ and $\tau$ denote, respectively, the shield outer radius, conductivity and wall thickness, where as $\boldsymbol{E}_{\boldsymbol{r}}$ and $\boldsymbol{b}$ indicate the relative permittivity and overall radius of the insulating covering. From the above equations

$-\quad=K_{v} \mathrm{~V}$

in which

$$
K_{v}=\frac{l_{c}}{4 a \sigma_{c} \tau} \sqrt{\frac{\varepsilon_{o} \varepsilon_{r c}}{10 \mu_{o} \ln (b / a)}}
$$

Here, $r_{0}$ and $r_{c}$ are the cable end ranges indicated in figure. 1

\section{RESULTS AND DISCUSSIONS}
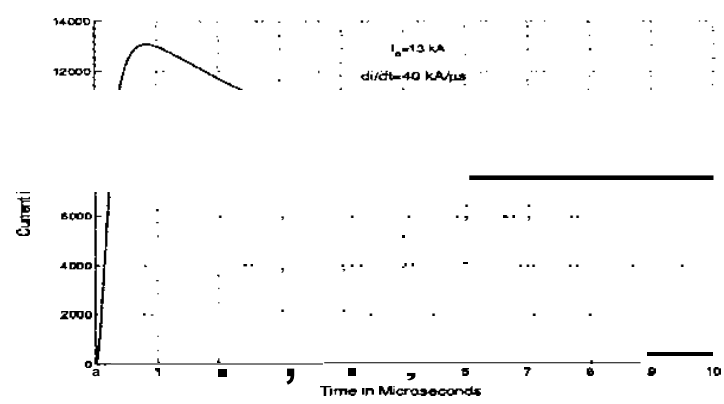

Figure 3. Current waveform chosen for computations

In the calculations, the return stroke channel current was chosen to be $13 \mathrm{kA}$ with maximum rate of rise of $40 \mathrm{kA} / \mu$ s. Figure 3 shows the current waveform used in the present study.

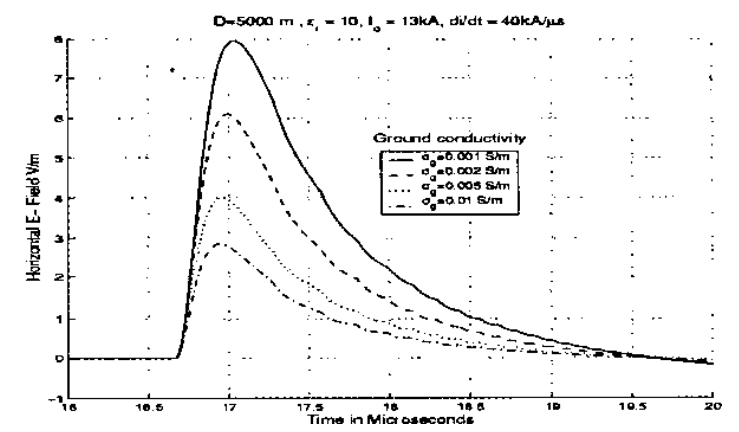

Figure 4. Horizontal electric fields at gmund surface $(s=0)$ for different ground conductivities.

Figures 4 shows the horizontal electric fields on the earths surface computed for various conductivities of the ground at a distance of $5000 \mathrm{~m}$ from the lightning channel. The permittivity of the ground $\boldsymbol{\varepsilon}_{\mathrm{r}}$, chosen is 
10. The peak values of the horizontal electric fields have also been shown. From the figures it is seen that the peak values and rise time of the horizontal electric fields increase with decreasing conductivities of the ground for a particular rate of rise of current. This is because of the absorption of high frequencies by the ground as the electromagnetic field propagates across it. The enhancement in the peak is generated by the larger wave tilt associated with decreasing conductivity.

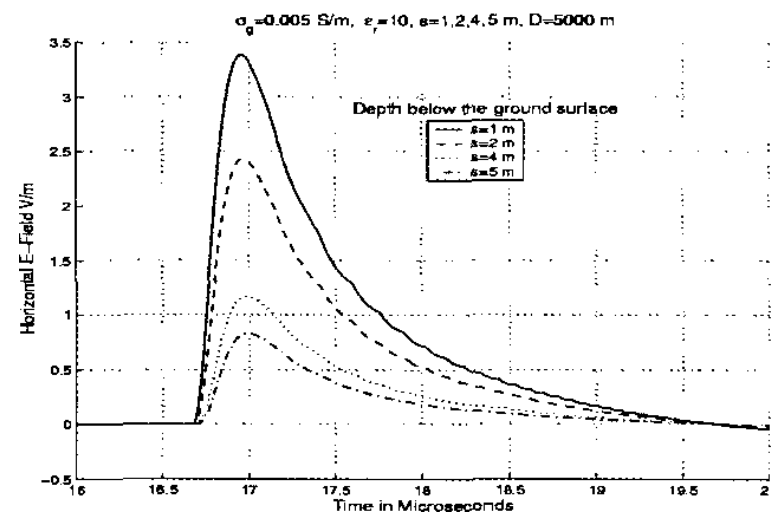

Figure 5. Horizontal electric fields at different depths below the ground surface

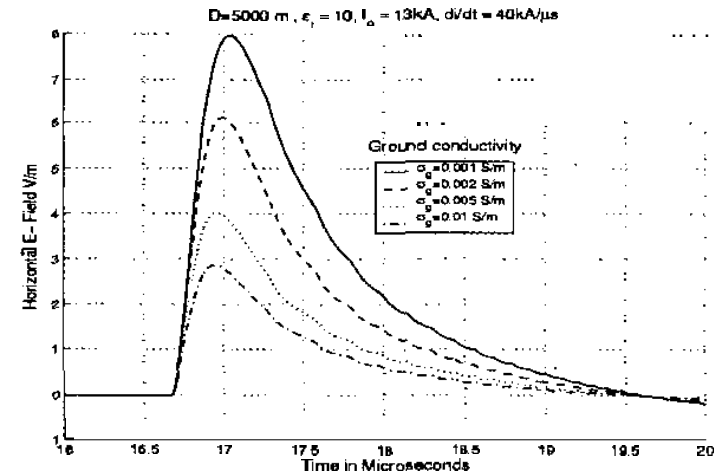

Figure 6. Horizontal electric fields at a depth of $1 \mathrm{~m}$ below the ground surface $(s=1)$ for different ground conductivities.

Figure 5 shows the horizontal electric fields at different depths below the ground surface. The results are shown for $s=1 \mathrm{~m}, 2 \mathrm{~m}, 4 \mathrm{~m}, 5 \mathrm{~m}$. The distance from the return stroke to the point of observation is $5 \mathrm{~km}$. The ground parameters chosen are $\sigma_{\mathrm{g}}=0.005 \mathrm{~S} / \mathrm{m}$ and $\varepsilon_{\mathrm{r}}=10$. From the figures it is seen that the peak values of the horizontal electric field decrease with increasing depth below the ground surface. Since the horizontal electric field is continuous across the boundary, unlike the vertical electric field it does not change in strength as one crosses it. But, as the horizontal electric field penetrates into the ground, the higher frequencies are selectively removed from it and, for this reason, the rise time of the the horizontal electric field increases with increasing depth for a particular value of the rate of rise of the current while its amplitude decreases.

Figure 6 shows the horizontal electric fields at a depth of $1 \mathrm{~m}$ below the ground surface for various conductivities of the ground at a distance of $5000 \mathrm{~m}$ from the lightning channel. The permittivity of the ground $\varepsilon_{r}$, is chosen as 10 . The peak values of the horizontal electric fields have been shown in figures. From the figures it is seen that the peak values and rise time of the horizontal electric field increase with decreasing conductivities of the ground. This is because of the absorption of high frequencies by the ground as the electromagnetic field propagates along it. The enhancement in the peak is generated by a larger wave tilt associated with decreasing conductivity.

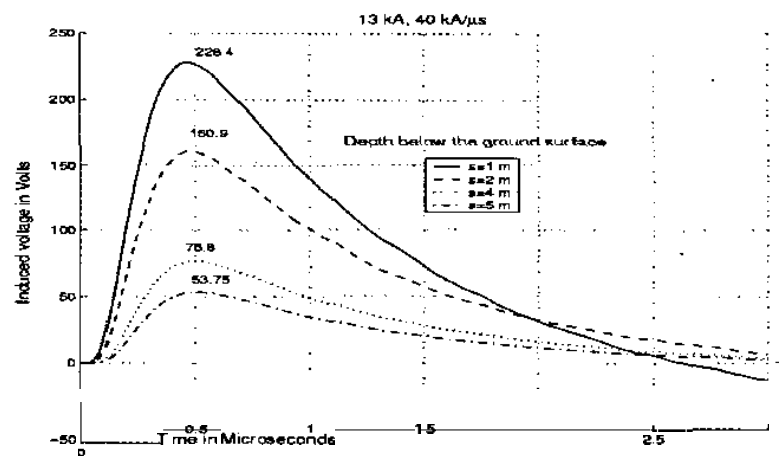

Figure 7 The induced voltages on the shielded cable buried at different depths below the ground surface

Figure 7 shows the induced voltages on the buried cable at different depths below the ground surface. The lightning strikes at $10 \mathrm{~m}$ away from one of the line terminations and perpendicular to the conductor. The ground parameters chosen are $\sigma_{\mathrm{g}}=0.005 \mathrm{~S} / \mathrm{m}$ $\varepsilon_{\mathrm{r}}=10.0$. The peak values of the induced voltage decreases with increase in depth because the horizontal component of the field decreases with the depth.

The induced voltages on the cable are computed for the lightning striking at $10 \mathrm{~m}$ away from one of the line terminations and perpendicular to the conductor at a depth of $1 \mathrm{~m}$ below the ground for different ground relative permittivities, viz., 5, 10 and 15 .The induced voltage on the cable at the termination is 
shown in figure 8. Since the ueak values of horizontal electric fields varies only marginally the same trend is reflected on the induced voltages. Hence it can be concluded that the variation in induced voltages due to variation in relative permittivity of ground is negligibly small for the range of values considered

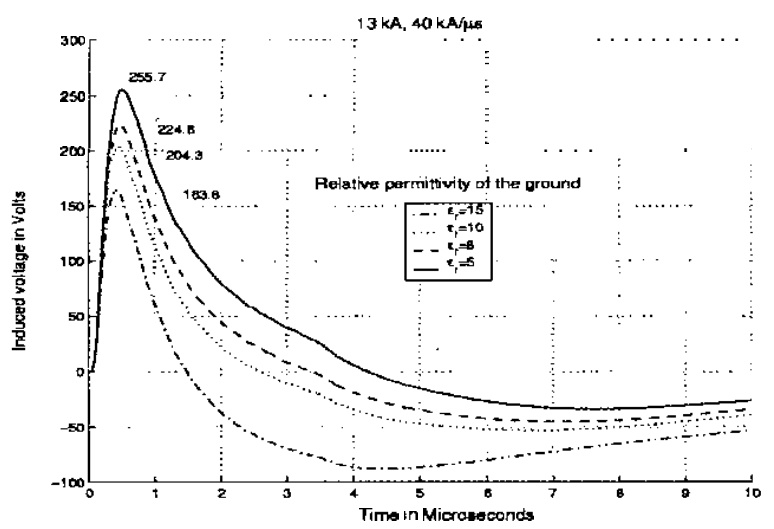

Figure 8. Induced voltages on the shielded cable buried at a depth of $1 \mathrm{~m}$ below the ground surface for different ground relative permittivities .

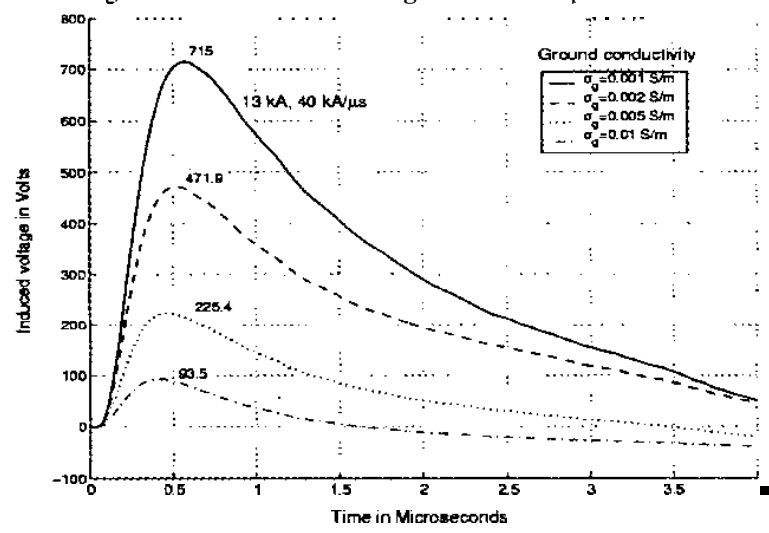

Figure 9. Induced voltages on the shielded cable buried at $1 \mathrm{~m}$ depth below the ground surface for different ground conductivities.

The induced voltages are computed on the cable for the lightning striking at $10 \mathrm{~m}$ away from one of the line terminations and perpendicular to the conductor at a depth of $1 \mathrm{~m}$ below the ground for ground conductivities of $0.01 \mathrm{~S} / \mathrm{m}, 0.005 \mathrm{~S} / \mathrm{m}, 0.002 \mathrm{~S} / \mathrm{m}$ and $0.001 \mathrm{~S} / \mathrm{m}$ keeping the relative permittivity of the ground constant at 10 . The induced voltage on the cable at the termination is shown in figure 9. From the figure it is seen that as the ground conductivity decreases the induced voltage peak decreases similar to that of the fields computed.

\section{CONCLUSION}

The radiated electric field due to a nearby lightning stroke has been computed at ground surface as well as at different depths below the ground surface. It has been seen that the horizontal electric field at the ground surface as well as below the ground surface reduces with increasing ground conductivity. The horizontal electric field also reduces with depth of the observation point below the ground surface.

The induced voltages on the buried cable due to the interaction of lightning electromagnetic field has also been computed. The induced voltage decreases with increasing depth of the cable below the ground where as it increases with decreasing ground conductivity. The permittivity of the ground also has a significant influence on the induced voltage.

\section{REFERENCES}

[1] M.J.Master and M.A.Uman,"Lightning induced voltages on power lines: Theory," IEEE Trans.Power App.Syst., Vol. PAS-103, 1984,pp.2502-2518.

[2] F.Heidler, "Travelling current source model for LEMP calculation", Proc. 6th Int.Symp. on EMC, Zurich, March 57, 1985.

[3] V.Cooray, "Horizontal fields generated by return strokes", Radio Science, Vol.27, No.4, July-August 1992, pp.529537.

[4] V.Cooray, "Underground electromagnetic fields generated by the retum strokes of lightning flashes", IEEE Trans.Electromag.Compat., Vol .43, No.1, February 2001, pp.75-84.

[5] Michel Aguet et al., "Transient electromagnetic field coupling to long shielded cables.", IEEE Trans.Electromag.Compat, Vol-EMC.22, No.4, November 1980,pp. 276-282.

[6] John D.Nordgard, Chin-Lin Chen, "Lightning induced transients on buried shielded transmission lines", IEEE Trans.Electromag.Compat., Vol-EMC.21, No.3, November 1979, pp. 171-181.

[7] V.Amorouso, F.Lattarulo, "Lightning -oriented tangential electric field across air-soil interfaces", IEE Proc.Sci.Meas.Technol., Vol.141, No.1, January 1994, pp.65-70.

[8] Vance, E.F., 'Coupling to shielded cables" WileyInterscience, New York, 1978. 